\title{
Delivery of Content for an Online Graduate Course on "The Management of Innovation for Engineers"
}

\author{
Harpreet Dhariwal \\ Instructional Technology Specialist \\ harpreet.dhariwal@utoronto.ca
}

Stephen Armstrong, P.Eng., M.A., FCGI, C.Eng., FIMechE, CMC

Adjunct Lecturer

stephenc.armstrong@utoronto.ca

Chris Damaren, PhD, PEng

Vice-Dean Graduate Studies

damaren@utias.utoronto.ca

\section{Faculty of Applied Science and Engineering University of Toronto}

\begin{abstract}
This paper will discuss the pedagogical impact and elaborate on the development and implementation of the first online graduate course at the Faculty of Applied Science and Engineering at the University of Toronto. The graduate course entitled "Management of Innovation in Engineering" (APS 1012H) provides students with the core concepts of innovation including; types of innovation, innovation diffusion, strategic and systems thinking, transformational change management, innovative enterprise design \& development, and sustaining a culture of innovation. The key course objectives are to expose engineers to real world strategic management challenges and facilitate them to think and write critically through experiential learning. This seminar style course was considered an ideal candidate for the initial pilot for the Faculty online delivery initiative. Both the course content and the course delivery methods were new initiatives at the university. This course incorporates both academic readings to provide the broad theory of innovation and in depth lectures and discussions based on the instructors many years of hands on practical experience in innovation in a variety of industry sectors. A team-based approach was taken to produce final project reports on a variety of topical innovation subjects. The development of a $100 \%$
\end{abstract}

online delivery was an iterative process spanning three academic terms. The course was first delivered to $100 \%$ in class students in the winter term 2010. In the fall term 2010 a hybrid approach was adopted were the course was delivered simultaneously to both in class and online students. The content from the inclass lectures was captured during live lectures using a sophisticated multimedia lecture capture system and delivered asynchronously to the online students. Great effort was made to ensure, the course objectives, content, evaluation, assessment, and academic rigour was equivalent for both sections. This first iteration provided valuable insight and revealed complexities of the online delivery specific to the course syllabus. The lessons learned were then used to refine and improve the online delivery and successfully teach a $100 \%$ online Engineering graduate course for the winter 2011 term. In summary, the challenge was to teach the management of innovation using a new innovative teaching process while maintaining the experiential learning objectives in a virtual environment.

\subsection{Introduction}

The Faculty of Applied Science and Engineering at the University of Toronto offers four graduate degrees: the Master of Engineering (MEng), the Master of Health Science in Clinical Engineering, the Master of Applied Science, and the Doctor of Philosophy. The MEng is a professional degree which may be taken in one of seven graduate units: Aerospace Science and Engineering, Biomedical Engineering, Chemical Engineering and Applied Chemistry, Civil Engineering, Electrical and Computer Engineering, Mechanical and Industrial Engineering, and Materials Science and Engineering. It may be taken part-time or full-time. In most units, the MEng degree requirements consist of ten courses with the exception of Electrical and Computer Engineering where it is nine courses. Historically, these course requirements were met by technical courses in the discipline under study.

Four years ago, the Faculty launched a new series of courses directed at MEng students in the areas of Entrepreneurship, Leadership, Innovation, and Technology in Engineering (ELITE). Example courses include Project Management, Financial 
Engineering, Human Resources Management, Concepts and Application of Authentic Leadership, and Management of Innovation in Engineering (APS1012H). Students who complete four courses from the ELITE area, receive an ELITE Certificate in addition to their degree. The ELITE courses have been very successful and many courses have enrolments exceeding 50 students.

For part-time MEng students who have daytime employment, it is difficult to attend courses during the day at the downtown St. George campus of the University of Toronto. For this reason, flexible delivery options have been created for some of the ELITE courses. In particular, some are offered on Saturday mornings, in the evenings during the week, and in a two-week intensive format during the summer months. Recently, the Faculty decided to embark on an online initiative where a pilot course would be offered in an online delivery mode. This would permit students to access the course content remotely at times that were suitable to them.

The course selected for the pilot was Management of Innovation in Engineering (APS1012H).

\subsection{Course Content}

\subsection{Setting the Stage - The Need}

"Science aims to understand nature and engineering, is about creating what has never been". Theodore Von Karmen

Innovation is the transformation of knowledge into products, processes, and services and is critical to economic competitiveness, long-term productivity growth, and wealth creation. Since the beginning of the industrial revolution in the $18^{\text {th }}$ century Engineers emerged as the key drivers of national and international innovation and quality of life. By the middle of the $19^{\text {th }}$ century engineering began to professionalize around functional disciplines that where designed around the underlying engineering science.[1] Currently powerful forces, including demographics, globalization, and rapidly evolving technologies are dramatically changing the nature of engineering practice demanding far broader more integrative skills than simply the mastery of scientific and technological disciplines. $21^{\text {st }}$ century challenges consist of complex system problems including transportation, communication, security, aging population, energy production and distribution, environmental remediation and sustainability. The $21^{\text {st }}$ century engineering profession must transition toward a model built on discovery, innovation and entrepreneurship [1]. This will require Engineers to move beyond the traditional foundation in pure sciences, mathematics, and engineering sciences to be drivers of strategic innovation and policy formation [1] - in a nutshell to "Engineer" strategic change.

The "Management of Innovation" course at the University of Toronto Faculty of Applied Science and Engineering provides students with the core concepts of innovation including; strategic thinking, transformational change management, innovative enterprise design \& development, and sustaining a culture of innovation. The management of innovation is interdisciplinary and multi - functional, requiring the alignment of market forces, technological systems and organizational change to improve the competitiveness and effectiveness of organizations and society. The process of innovation management is essentially generic, although organization, technological and market specific factors will constrain choices and actions [2]. This course incorporates both academic readings to provide the broad theory of innovation but most of the readings and discussion will be based on the instructors many years of hands on practical experience in the management of innovation in a variety of industry sectors.

\subsection{Course Outcomes}

While the course cannot train students in the detailed competencies to operate as expert innovators at a senior consultant level, it will raise their awareness on the broad issues that must be addressed to build and sustain an innovative enterprise and think strategically. Another graduate course has been designed to develop detailed competencies.

APS1012 is designed for students to enter a self directed discovery process. Traditional engineering courses have a tendency to be unidirectional and lecture centric. This course is heavily focused on enquiry and critical thinking. The expected outcomes from the course are:

- Establish a context for Innovation and its dimensions

- Understand how to analyze the elements of Innovation management as a key organizational capability

- Explain how innovations are diffused across cultures

- Understand how to develop an innovative future state vision through systems thinking 
- Define the preparation required for an organization to embark on a innovation transformation initiative

- Explain and assess a culture of innovation for readiness to change

- Identify the methods to overcome the barriers to innovation in organizations

- Develop the key leadership roles in managing innovation at the group/department, division, and corporate levels

- Develop an integrated enterprise approach to innovation

- Design an Innovation delivery system for various environments

- Apply process management and project management practices to product development

- Organize and develop integrated Project / Product teams (IPT's) for project execution

- Deploy and Embed an innovation process through the core culture

- Continuously assess the "As Is" business processes

- Continuously improve the As Is process through structured problem solving

- Apply the key strategies to engage the people to sustain an innovative culture

- Identify challenges in measuring and maintaining innovation performance

\subsection{Course Structure}

The Managementof Innovation course is divided into four themes and 12 modules as follows:

- The first theme establishes a context for Innovation that includes numerous case histories of companies managing certain types of technological change. We discuss various kinds of innovation and innovation diffusion through the ages.

- $\quad$ The second theme is; Design andDeveloping an Innovative Enterprise. The sources of success for great companies lie in what they do very well. The business model that has created great success is a barrier to change, as well as a source of advantage. Disruptive Technologies fundamentally challenge the company's current state business model which is why they are so disruptive. We discuss the approach to transformational change including visioning, process management, overcoming resistance to change, systems thinking, and effective teams
- The third theme examines; Building the Innovation Management Process. Once we understand how to overcome the barriers to change, we must then find out how companies can organize to achieve an innovative culture. We examine how to design an innovative process management system that allows a company to design and build products more effectively and launch new products effectively. We then examine enterprise processes in variety of industry sectors (Aerospace -Military and Commercial, Food, Machinery, Newspapers). We will examine the integration of standard project management techniques with process management.

- The fourth theme considers; Sustaining the Innovative Lean Enterprise. Once we understand how to overcome the barriers to change, we must then find out how companies can sustain an innovative culture. We will examine how to continuously analyze and improve business processes and business models. Central to achieving this culture is to embed systematic problem solving at the heart of day-to-day operational activities.

\subsection{Course Module Design}

The course was structured around the typical $12-13$ week semester normal in Canadian Universities. Each module consisted of a variety of readings that included chapters from the instructor's published books [2] [3] white papers, from, and academic scholarly papers.

Part 1 - Establishing a Context for Innovation

Week 1 - Orientation, instructor background, syllabus overview, \& assignments

Week 2 - Dimensions of Innovation

Week 3 - Diffusion of Innovation

Part 2 - Developing an Innovative Enterprise

Week 4: - Designing and developing an Innovative Lean Enterprise through Business Transformation

Week 5 - Organizational Approach to Cultural Transformation

Part 3 - Building the Lean Innovation Management Process

Week 6 - Enterprise Integration and Process

Week 7 - Integrating Process, Project, Programme Management \&Integrated Project Team's

Week 8 - Lean Product Development and Knowledge Management

Week 9 - Deploying and embedding the innovation process 
Part 4 - Sustaining the Innovative Lean Enterprise Week 10 - Continuous Analyses of the "As Is" / "To Be" Business Process

Week 11 -Continuous Improvement through Structured Problem Solving

Week 12 - Maintaining an Innovative Culture. Also Final Draft Report Team Presentation

Week 13 - Project Presentations - Online Video

The course was taught through a combination of formal 45-60 minutes classroom lectures, critical review presentations, and student class participation.

A total of 3 critical review readings were required from each student. Critical reviews consisted of readings from each theme. The student had available to them a variety of choices from two eBooks on innovation and over 100 scholarly articles. Emphasizes was placed on thinking critically and sample reviews and guides were provided.

Final Paper-Project Report: The students were formed into teams and produced a group project report. The goal of the final project report was not to do original field research, but to demonstrate the ability to apply innovation concepts in a situation of their choosing.

\subsection{Moving From Physical to Virtual and $100 \%$ Online Delivery}

\subsection{Process (Three Phases to Online Delivery)}

The graduate course entitled "Management of Innovation in Engineering" (APS 1012H) described above in detail above in section 2 was considered an ideal candidate for the initial pilot for the Faculty of Applied Science and Engineering online course delivery initiative. Both the course content and the course delivery methods were new initiatives at the University of Toronto.

The challenge for the online delivery was to ensure, the course objectives, the course content, the evaluation, the course assessment, and the academic rigour was equivalent to the physical in class traditional course.

The path to a $100 \%$ online course delivery was an evolutionary three phase approach as follows:

Phase 1:100\% Physical and traditional in class lectures.

Phase 2: Combination of traditional in class and online delivery (Hybrid approach).

Phase 3: 100\% virtual online delivery.

\subsection{Phase 1, Inaugural Delivery}

The course content was originally developed in 2009 for a traditional seminar style graduate course and was first delivered in the winter 2010 term (January 2010).

The course was initially designed for an in-class experience with no thought of an online course delivery option. Upon completion of the inaugural course in April 2010 a standard University of Toronto course evaluation survey was conducted and feedback from 32 students was received. The standard evaluation method provided consistent method for eliciting feedback from the students.

The course evaluation was designed around two elements - the course content and instructor performance. Both course content and the instructor received good ratings (course content $92 \%$ and for instructor $94 \%$ )

Based on this positive student feedback on the initial delivery of the "Management of Innovation" course, the course content and the instructor abilities to deliver modular content, a Faculty decision was made to attempt to deliver the course content to an online audience.

Traditional engineering courses require 'chalk and talk' as a method of delivery and therefore can be a difficult to deliver to an online virtual audience [4].

As Carrol [4] states, "The chalkboard is a very powerful tool for teaching engineering mechanics courses. The strengths of the chalkboard are that it is a very versatile tool, it is very reliable, it is inexpensive, and it doesn't require the faculty member to learn a complex software package"

The modular non-technical course content for the "Management of Innovation in Engineering" (APS $1012 \mathrm{H}$ ) and the fact that the course content was predominantly delivered by static PowerPoint made it ideal for an attempt to covert the course content and redesign the course to be available in an virtual online environment.

\subsection{Phase 2, Hybrid Approach: Online and In Class}

After the initial success of the physical in class course delivered in the winter term 2010 (success based on the formal student evaluation and informal feed back from the instructor), in the fall term 2010 a hybrid delivery approach was adopted. In September 2010 the Management of Innovation in Engineering (APS 
$1012 \mathrm{H})$ course was delivered simultaneously to both in class and online students.

In the fall of 2010, for the first time in the history of the Faculty an online section was established in the official University student information system ROSI (Repository Of Student Information) for an Engineering graduate course.

Since this was a new and novel approach to course delivery, to manage the online course expectations from the online students and the instructor the registration for the first online course offering was capped at 10 . Remarkably, these 10 spots were filled within the first 45 minutes of opening the registration to the students.

\subsection{Online course structure}

The online course was structured to be transparent to the successful teaching style of the instructor. Each course module remained the same as the physical in class section (consisting of, readings, white papers, chapters from the instructors published books, and academic scholarly papers.)

The only difference in this phase 2 was that the course modules were published each week on the University learning management system. Each published online courses module consisted of module specific content and a distinct virtual discussion board linked for each module. The discussion board was optional for in class students and made mandatory for the online students.

This was accomplished by offering class participation marks to the online students based on their overall virtual participation in the online discussion.

Individual modules were designed for the benefit of the online students and were also used by the in class students to complement the weekly lectures.

\subsection{Capture Technology and Online Delivery}

The content from each weekly in-class lectures was captured during the live in class lectures to the physical students using a sophisticated multimedia lecture capture system. The lecture capture system consisted of a high definition video camera, a wireless microphone system and a complex lecture capture appliance to record and synchronizes the PowerPoint slides.

The objective of deploying a sophisticated lecture capture system was to deliver modular course content asynchronously to the online students and to ensure that the online students received exactly the same level and quality of instruction as the physical class. Please see "Figure 1. Screen Capture of Lecture Capture" for an example of the end result technology.

In addition to offering quality instruction, lecture capture allows flexibility in learning and accommodates the many different learning styles for engineering students. [5]

The screen capture image below "Figure 2. Example of entire lecture view" demonstrates the ability of the online student to watch the captured lectures in their entirety or partially by skipping to any necessary PowerPoint slide in the online lecture. "

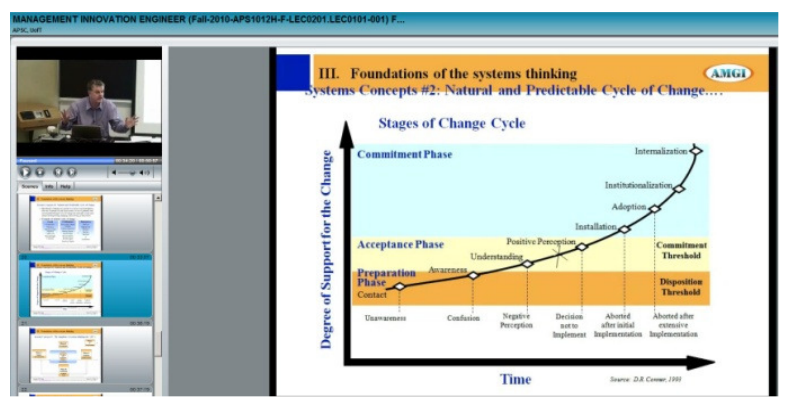

Figure 1. Screen Capture of Lecture Capture

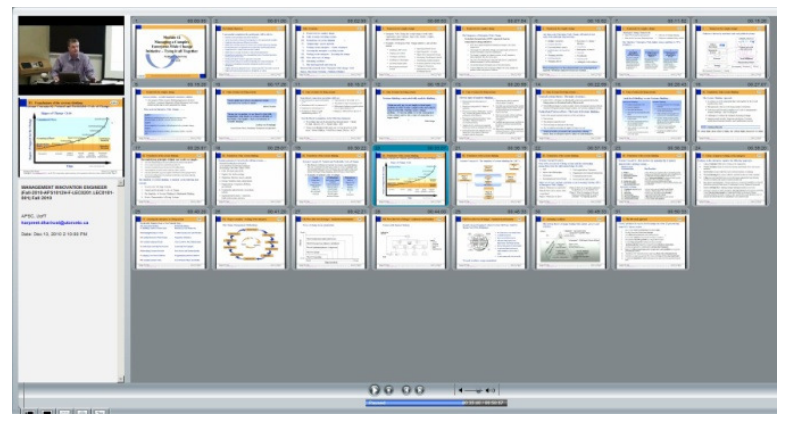

Figure 2. Example of entire lecture view

In this hybrid model, class sessions were offered both online and in the physical classroom so that students could attend either or both-those who attend face-to face sessions could join the online class discussion, and those who attend online could later review the inclass sessions that were posted in audio or audio/video format. If distant and time permitted some online students on occasion decided to attend the in class lectures and conversely some in class students went $100 \%$ online. This occurred for various reason either because some students were used to live lectures and required the live face to face contact and found the 
live contact more stimulating. Other students switched to $\% 100$ online because of the convenience and geography independence.

This first iteration of online delivery provided valuable insight and revealed complexities of the online delivery technology. For example, the authors learned through feedback from the online students that the successful deployment of the Hybrid model is highly dependent on the online technology tools and the quality and timely release of course content including the captured lectures.

The online students expressed frustration for performance or availability issues with the learning management system or if technology caused delays between the capture of the in class sessions and their availability to students. In addition some online students expected synchronous instructor feedback on the virtual discussion.

We believe this frustration with online delivery was reflected in the course assessment. In phase 2 the hybrid model course evaluations the physical in class students marked the class and the instructor in the high 80 's but the online student evaluation marks were in the high 70's. We believe the assessment results reflect the challenge of mixing online with in class and the online learning curve.

\subsection{Phase 3, Evolution and Deployment of $100 \%$ Online Delivery}

For the winter 2011 term, the lessons learned from the hybrid model course delivery in phase 2 above were used to refine and improve the online delivery and successfully teach a $100 \%$ online Engineering graduate course.

Using the valuable lessons from the hybrid approach and the student feedback from the course evaluations the "mechanics" of the online delivery were improved with more streamlined and user ready processes. For example by repurposing the video lectures from the fall 2010 hybrid class the authors were able to maintain a more structured and timely video release schedule each week. In addition, we established a code of conduct that established online student expectations right from the outset.

\subsection{Expectations of Online Students}

Base on the experience of the first online hybrid offering in September 201 the January 2011 online students were provided a list of expectations including the statement at the outset of the online course, that $\% 100$ Online learning requires as much or more effort than in class learning. Although it is perceived that $\% 100$ online students may have several advantages to the learning process such as the ability to engage in learning asynchronously at a time and a place that is convenient, it was impressed on the January 2011 online students that the academic rigor and learning outcomes would be identical to an in class experience.

The following baseline rules for successful online learning were presented to the first $100 \%$ online class in January 2011:

Online Students:

- Must understand that participation in the virtual discussions board is as rigorous as any physical seminar style graduate course - the same level of originality and critical thinking was required and assessed.

- Must commit and engage in online discussion from the end of the first week. Marks for online discussion are evenly distributed over the 12 modules.

- Must read and understand the student performance evaluation rubric and demonstrate this by discussing online how it works by the end of the 2 nd week.

- Must provide a 2-minute background introduction video by the end of the 2 nd week.

- Will be expected to produce 3 videos - 2 for verbal critical review presentations and 1 final project presentation.

- Understand a lack of engagement in the discussion board evenly paced throughout the course will result in penalties from a grade viewpoint. Each lecture will be posted for 1 full week and shut off permanently the week of the 2nd lecture. This means online discussion on the lecture must occur prior to the next lecture.

\subsection{Online Delivery and Lessons Learned}

The iterative development of a $100 \%$ online delivery, the first for the Faculty of Applied Science and Engineering at the University of Toronto, spanned three academic terms. The various iterations and phases provided important evolutionary steps for successful online course delivery while maintaining the equivalent academic rigour as a physical in class graduate engineering courses. 
The opportunity to teach the management of innovation using an innovative online teaching process provided the authors exposure to new teaching technologies for delivering content and for online student engagement. We believe effectively engaging online students in the virtual space is fundamental to the success of the online course and share below several strategise developed and successfully deployed in the first $100 \%$ online course in the Faculty:

Design activities around grades. This is by far the most important lesson. In the hybrid model we found it very difficult to get the online students to engage because we were still thinking like the in class model. By assigning $30 \%$ of the marks to online discussion and 2.5 points to each discussion students engaged. We provided a rubric that specified precisely what was considered good versus mediocre engagement. For example advancing a discussion and introducing new concepts received additional marks.

The best discussions are "reality-based" activities. The more a student can relate to a learning activity, the easier it is for that student to get involved in it-and employ the purpose of the activity beyond the course. We created discussion questions that centred on the theory discussed in the lecture but encouraged students to relate the ideas to their work experience or other case studies

Be on the lookout for existing or potential media sources that can be morphed to fit your class. From textbooks that include activities to you tube presentations, to daily events to newspaper and magazine articles to conferences and conversations to blogs to TV shows and movies to everyday life experiences - each of these (and others) presents you with a gold mine from which to unearth new and exciting activities for your course.

\section{Provide Prompt Feedback}

Lack of personal contact can be a quality concern online. Providing regular feedback can help this. It does take considerable time to respond to student emails. The best way to avoid having to respond to each email to make students post their issues as long as they are not to personal on the discussion board. An online instructor may have to set limits on how much time s/he can devote to communications.

Students need e-mail guidelines at the beginning of a course.

Keep all discussions short and easy to understand. Online discussions are not meant to be treatises, dissertations, essays, or theses; they must be short to hold students' attention and to fit as a component of a larger lesson. Encourage students to respond to instructor assigned questions but more importantly encourage students to respond to one another.
Use humour in discussions. Humour is always a good method to engage students and to make the learning experience less intense. Discussions that evoke a smile, or chuckle are effective as they have a friendly flavour, thus making them easier and more enjoyable to tackle. It is much easier to use humour in in-class sessions where one can judge the response in realtime. But observe three notes of caution. (1) Use these sparingly online, not as the norm, for you want a balance in the style of your activities (too much humour can take away from the serious nature of the course). (2) Be sure it is humorous. University of Toronto engineering courses attract students from all over the world with cultural sensitivities. Too many people try their hand at humour and fail miserably because they forget the multi cultural environment of the students. (3) always keep the humour in good taste for complex cultures in the class. And if this seems a daunting task avoid humour.

Make use of the newest online technology. Beyond discussion board and text, there are blogs, podcasts, videos, animation, .mps (audio) files, interactive links, and other options-explore these and see which ones might be a nice bonus to an activity in engaging your students, in presenting your activity's purpose, and in spicing up your class.

\section{References}

[1] Mokyr, J, The British Industrial Revolution: An Economic Perspective Westview Press, Boulder, CO, 1999

[2] Armstrong SC; Sustaining Innovation through problem solving Industrial Press New York, 2008

[3] Armstrong, SC, Engineering Product and Development Management the Holistic Approach Cambridge University Press, Cambridge, UK 2001

[4] Carroll and Hong Sheng, "Incorporating Technology into the Traditional Engineering Mechanics Lecture", ASEE 2007 Southwest Section Conference, University of Nevada, April 12-13, 2007

[5] Cleghorn, W.L. and Dhariwal, H., "Impact of Online Lectures for an Undergraduate Mechanisms Course" ASEE 2011 Northeast Section Conference, University of Hartford, April 29-30, 2011 